\title{
Neuroendokrine pulmonale Tumore: verbesserte Diagnose im DOTATOC- versus FDG-PET/CT
}

\author{
Neuroendocrine Pulmonary Neoplasms: Improved Diagnosis by \\ DOTATOC- vs. FDG-PET/CT
}

Autoren

Institute
D. Frechen ${ }^{1}$, Y. Temur ${ }^{2}$, C. Cornelissen ${ }^{1}$, S. Kweider ${ }^{1}$, S. Krüger ${ }^{1}$

${ }^{1}$ Medizinische Klinik I, Universitätsklinikum, RWTH Aachen

${ }^{2}$ Klinik für Nuklearmedizin, Universitätsklinikum, RWTH Aachen eingereicht $\quad$ 18.08. 2011 akzeptiert nach Revision 18. 08. 2011

Bibliografie

Dol http://dx.doi.org/

10.1055/s-0031-1286634

Pneumologie 2011; 65: 628-630

(c) Georg Thieme Verlag KG

Stuttgart · New York

ISSN 0934-8387

Korrespondenzadresse Dr. med. Dirk Frechen

Medizinische Klinik I

Universitätsklinikum, RWTH

Aachen

Pauwelsstr. 30

52074 Aachen

dfrechen@ukaachen.de
Ein 56-jähriger Patient stellte sich mit rezidivierenden Palpitationen bei seinem Hausarzt vor. Ursächlich für die Palpitationen war paroxysmales Vorhofflimmern. In einer kardialen Computertomografie zeigte sich kein Hinweis auf koronare Verkalkungen, jedoch nebenbefundlich ein Rundherd im linken Lungenunterlappen.

Zur weiteren Abklärung des malignomsuspekten Rundherdes erfolgte die Vorstellung in unserer Klinik. Bis auf die Palpitationen war der Patient subjektiv beschwerdefrei und im Alltag gut belastbar. Fieber, Nachtschweiß und ungewollter Gewichtsverlust wurden verneint. Es bestanden keine relevanten Vorerkrankungen und keine Dauermedikation. Der Patient war Nichtraucher. Die Familien- und Berufsanamnese waren ohne Besonderheiten. Der körperliche Untersuchungsbefund des Patienten in gutem Allgemeinzustand war bis auf eine leichte Adipositas (BMI $31 \mathrm{~kg} / \mathrm{m}^{2}$ ) unauffällig. Eine kapilläre Blutgasanalyse und die Lungenfunktionstestung erbrachten normale Werte.

Zur Ausbreitungsdiagnostik des malignomsuspekten Tumors im Lungenunterlappen links erfolgte die Durchführung einer Positronen-Emissions-Tomografie, kombiniert mit einer Computertomografie (PET/CT). Standardmäßig wurde als Radiomarker für das PET/CT ${ }^{18} \mathrm{~F}$-Fluorodeoxyglukose (FDG) verwendet. Der Rundherd im linken Lungenunterlappen zeigte nur eine geringe Speicherung des Radiomarkers mit einem maximalen ,Standardized Uptake Value' (SUVmax) von 3,1 und nach peripher angrenzend eine diffuse flächige geringgradige Speicherung. Zusätzlich bestand eine geringe bandförmige Speicherung in der 5. Rippe rechts anterior (SUVmax 2,5). Kräftigere fokale Speicherungen stellten sich in Projektion auf eine Sklerosierung in LWK1 links anterior (SUVmax 6) und auf das Os sacrum rechts kranial in der Nähe des Iliosakralgelenks (SUVmax 5,1) dar.
Bronchoskopisch wurden Biopsate aus dem Lungenrundherd entnommen. Die Histologie ergab die Manifestation eines hochdifferenzierten Tumors. Immunhistochemisch wurde bei Negativität für TTF-1, CK7 und CK20, aber starker Positivität für Chromogranin und Synaptophysin die Diagnose eines hochdifferenzierten neuroendokrinen Karzinoms gestellt.

Aufgrund dieses Befundes erfolgte ergänzend zur Ausbreitungsdiagnostik ein PET/CT mit ${ }^{68} \mathrm{Ga}-\mathrm{DO}-$ TATOC. Bereits im Übersichtsbild der DOTATOCPET reicherten, verglichen mit dem FDG-PET, mehrere Läsionen deutlich kräftiger an $(\bullet$ Abb. 1). Die pulmonale Raumforderung links zeigte nun eine malignomtypische starke DOTATOC-Anreicherung (SUVmax 16,5). Im Bereich der 5. Rippe rechts, wo sich eine schwache Glukosemehranreicherung im FDG-PET/CT fand, bestand eine sehr kräftige fokale DOTATOC-Speicherung (SUVmax 12,9). Eine multiple ossäre Metastasierung in der 5. Rippe rechts, LWK1 (SUVmax 30,4) und im Os sacrum (SUVmax 23,7) kann aufgrund des DOTATOC-PET/CT als gesichert angenommen werden. FDG- und DOTATOC-PET vergleichend, sind exemplarisch Bilder in Abb. 2 dargestellt.

Die FDG-PET/CT ist in der Pneumologie zur Beurteilung der Dignität von pulmonalen Rundherden und dem präoperativen Staging des nichtkleinzelligen Bronchialkarzinoms mittlerweile weit verbreitet. FDG als Marker des Glukosemetabolismus reichert in Malignomen mit hohem Glukosestoffwechsel, aber auch in Organen wie Leber, Herz, Darm oder Gehirn an und erlaubt hier nur eine eingeschränkte Beurteilung. Langsam proliferierende Karzinome können daher keine oder nur eine geringe FDG-Aufnahme zeigen. Die FDGPET/CT hat für diese Malignome nur eine geringe Sensitivität. In den letzten Jahren wurden neue Radiopharmazeutika mit molekularen Zielstrukturen wie zum Beispiel zelluläre Rezeptoren oder Oberflächenantigene entwickelt [1]. DOTATOC ist eines der Radiopharmazeutika dieser neuen Ge- 


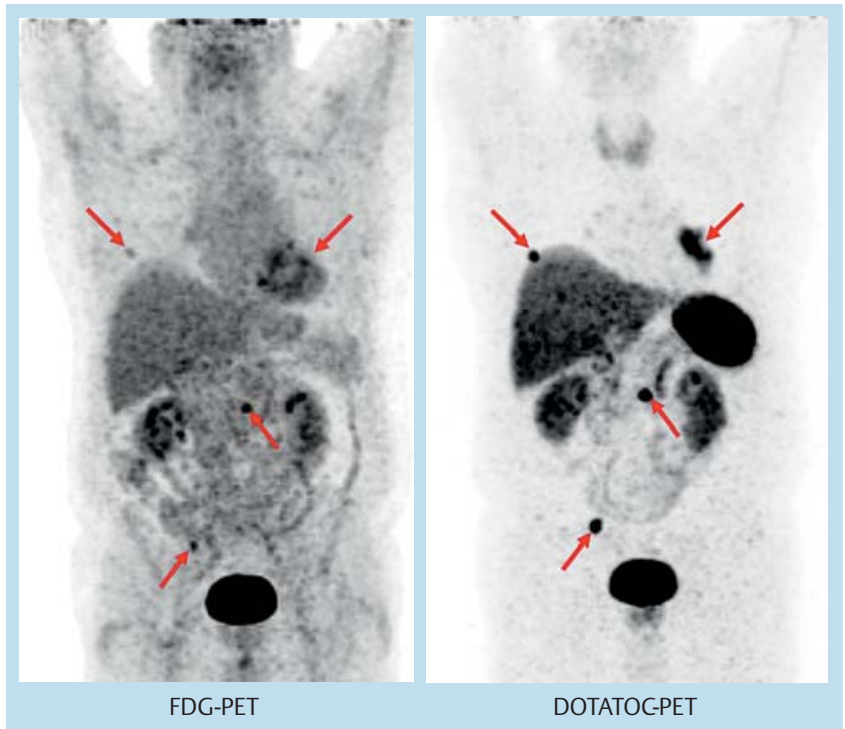

Abb. 1 Vergleich des FDG-PET (links) mit dem DOTATOC-PET (rechts) eines Patienten mit einem metastasierten neuroendokrinen Tumor; pfeilmarkiert sind die Tumormanifestationen.

neration und setzt sich aus dem Komplexbildner 1,4,7,10-Tetraazacyclododecan-N',N",N"',N"'--Tetraessigsäure (DOTA) und dem Octreotid-Analogon [D]-Phe ${ }^{1}-\mathrm{Tyr}^{3}$-Octreotid (TOC) zusammen. DOTATOC bindet an den Somatostatinrezeptor 2 und kann mit ${ }^{111} \mathrm{In},{ }^{68} \mathrm{Ga}$ oder ${ }^{86} \mathrm{Y}$ für diagnostische Anwendungen und mit ${ }^{90} \mathrm{Y}$ für therapeutische Anwendungen gebunden werden [2]. $\bullet$ Tab. 1 stellt FDG und DOTATOC vergleichend gegenüber.

Neuroendokrine Tumoren (NET) sind hochdifferenzierte Tumore und exprimieren zahlreiche Rezeptoren für spezifische Amine
Tab. 1 Charakteristika des FDG und DOTATOC als Marker in der PET/CT.

\begin{tabular}{|c|c|c|}
\hline & FDG & DOTATOC \\
\hline Ligand & Fluorodeoxyglukose & $\begin{array}{l}\text { Komplexbildner 1,4,7,10- } \\
\text { Tetraazacyclododecan- } \\
\text { N',N",N"',N"','-Tetraes- } \\
\text { sigsäure (DOTA) und } \\
\text { Octreotid-Analogon [D]- } \\
\text { Phe'-Tyr'-Octreotid (TOC) }\end{array}$ \\
\hline markiert & Glukosemetabolismus & Somatostatinrezeptor 2 \\
\hline $\begin{array}{l}\text { Ausbreitungs- } \\
\text { diagnostik von }\end{array}$ & $\begin{array}{l}\text { Malignomen mit hohem } \\
\text { Glukosemetabolismus }\end{array}$ & $\begin{array}{l}\text { Neuroendokrinen Tumoren } \\
\text { und differenzierten Schild- } \\
\text { drüsentumoren }\end{array}$ \\
\hline Limitationen & $\begin{array}{l}\text { - physiologisch hohe } \\
\text { FDG-Aufnahme im } \\
\text { Gehirn, Myokard, } \\
\text { Leber und Darm } \\
\text { - eingeschränkte Diffe- } \\
\text { renzierung zwischen } \\
\text { posttherapeutischer } \\
\text { Inflammation und } \\
\text { Tumor } \\
\text { - geringe Sensitivität für } \\
\text { langsam wachsende } \\
\text { Tumoren }\end{array}$ & $\begin{array}{l}\text { Expression von Somato- } \\
\text { statinrezeptoren auf Ziel- } \\
\text { strukturen erforderlich }\end{array}$ \\
\hline
\end{tabular}

und Peptide wie zum Beispiel Somatostatin [3]. Ein spezifischer Ligand der Somatostatinrezeptoren wie DOTATOC lässt, verglichen mit FDG, eine höhere Sensitivität in der Ausbreitungsdiagnostik von NET erwarten. In einem Fallbericht ermöglicht DOTATOC im Gegensatz zum FDG eine Unterscheidung von differenziertem und undifferenziertem Tumorgewebe eines NET [4]. Von 63 Läsionen eines metastasierten NET bei 15 Patienten zeigten 43 (68\%) eine erhöhte FDG-Aufnahme, während sich 57 (90\%) mittels DOTATOC darstellten [5].

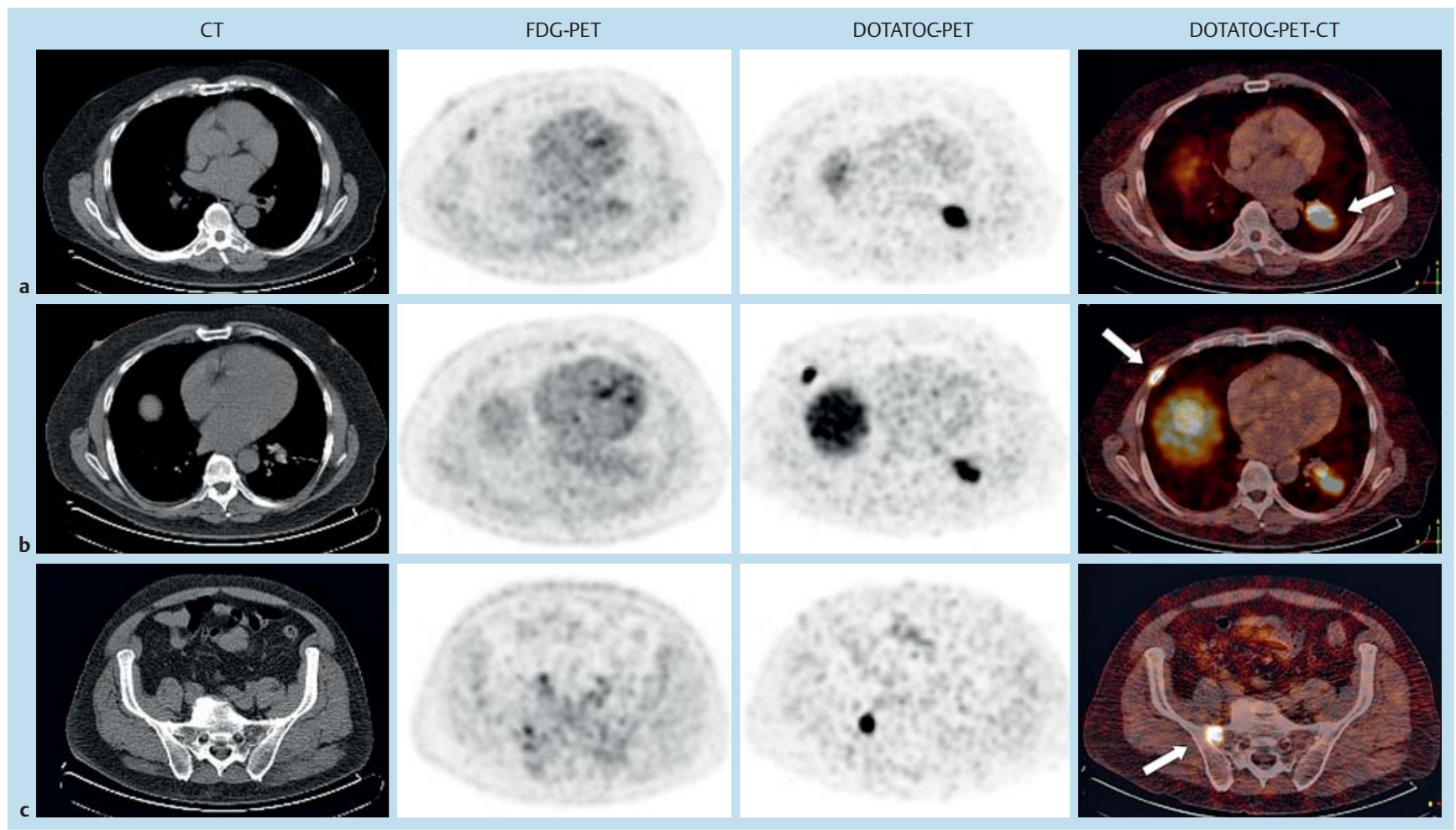

Abb.2 Von links nach rechts: Computertomografie, FDG-PET, DOTATOC-PET, Fusionsbild DOTATOC-PET/CT. Pfeil: Manifestationen des neuroendokrinen Tumors; a im linken Lungenunterlappen, $\mathbf{b}$ in der 5. Rippe rechts, c Os sacrum. 


\section{Fazit für die Praxis}

Bei pulmonalen neuroendokrinen Tumoren ist das DOTATOCdem FDG-PET/CT zur Ausbreitungsdiagnostik neuroendokriner Tumoren überlegen. Durch die sensitivere Diagnostik lässt sich die erforderliche Therapie besser planen.

\section{Interessenkonflikt}

$\nabla$

Die Autoren geben an, dass kein Interessenkonflikt besteht.

\section{Literatur}

1 Rice SL, Roney CA, Daumar $P$ et al. The next generation of positron emission tomography radiopharmaceuticals in oncology. Semin Nucl Med 2011; 41: 265-282

2 Heppeler A, Froidevaux S, Eberle AN et al. Receptor targeting for tumor localisation and therapy with radiopeptides. Curr Med Chem 2000; 7 : 971-994

3 Kaltsas GA, Besser GM, Grossman AB. The diagnosis and medical management of advanced neuroendocrine tumors. Endocr Rev 2004; 25 : $458-511$

4 von Falck C, Boerner AR, Galanski $M$ et al. Neuroendocrine tumour of the mediastinum: fusion of 18F-FDG and 68Ga-DOTATOC PET/CT datasets demonstrates different degrees of differentiation. Eur J Nucl Med Mol Imaging 2007; 34: 812-812

5 Koukouraki S, Strauss LG, Georgoulias V et al. Comparison of the pharmacokinetics of 68Ga-DOTATOC and [18F]FDG in patients with metastatic neuroendocrine tumours scheduled for 90Y-DOTATOC therapy. Eur J Nucl Med Mol Imaging 2006; 33: 1115-1122 